\title{
COMPUTATIONAL FATIGUE DAMAGE PREDICTION OF THE LORRY FRAMES UNDER RANDOM EXCITATION
}

The paper deals with FEM application in the TATRA 815 lorry frame construction fatigue life estimation. Construction of critical nodes from the fatigue damage point of view was found and analyzed. Analysis of the particular traffic state influence on the fatigue damage cumulation will be presented too.

Keywords: computer simulation, random vibrations, finite element method, fatigue life, cumulative damage prediction

\section{Introduction}

The process of fatigue damage (fatigue life) prediction is generally very complicated and contains a lot of uncertain inputs, e.g. material characteristics, randomness of the service loading etc. Our paper aim is to present the selected results of the computer simulation analysis of fatigue damage lorry frames. This simulation concentrates to:

- the most important aspects which are typical for means of transport working conditions,

- use of the obtained computational models for a working exciting simulation of a virtual lorry construction,

- dynamic analysis of the vehicle critical parts stress under the influence of typical working conditions,

- the fatigue life prediction of the analyzed vehicle most exposed parts.

During the computational simulation of the chosen vehicle (TATRA $815 \mathrm{~S} 1$ ) under the modeled conditions of its service, it was necessary to consider that it is a kind of vehicle whose traffic conditions are determined mainly by the influence of the following aspects:

- roadways and terrain surface undulation,

- traffic velocity.

\section{Analyzed Vehicle Characterization}

It is known from technical publications and from similar performed analyses of various kinds of transportation vehicles, that the most loaded lorry parts are their bearing members (bearing frames, subsidiary bearing constructions) and axles. They carry the loads occurring at the interaction of the vehicle instantaneous weight influence and roadways surface undulation influence in synergy with chosen traffic velocity.
This is the reason why the presented description is oriented mainly to these construction entities. TATRA $815 \mathrm{~S} 1$ lorry undercarriage consists of a frame, a subsidiary frame, front and back hangers composed of axels with wheels, cushioning, own brakes and an axle control operating machinery, control and brakes machinery.

The main vehicle bearing part is formed by a spinal frame to which a subsidiary ladder frame for bodywork assessment, a drivinggear and a sleigh is mounted. The spinal frame consists of particular axle gear-boxes, an additional gear-box, front and back joining piece, front and back bearing tube and transoms. The connection of the mentioned parts is realized by means of flanges and bolted connections.
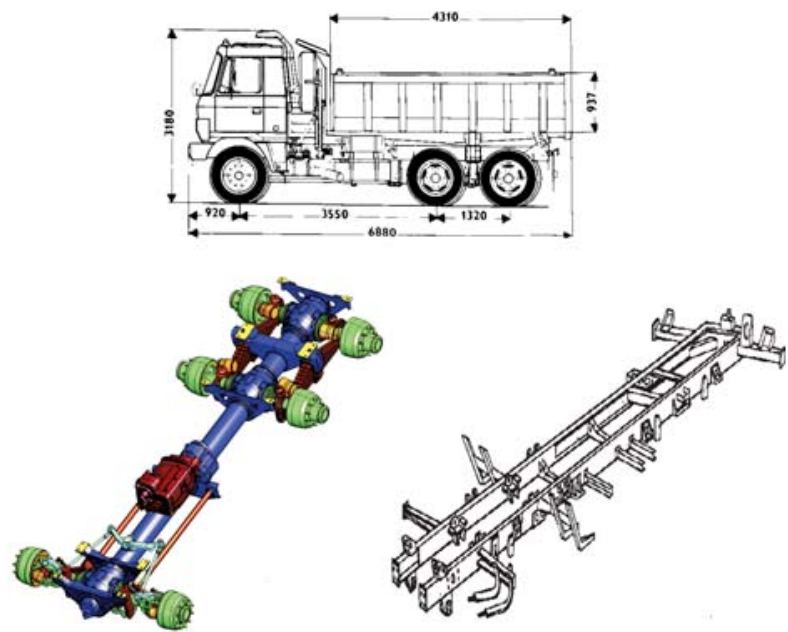

Fig. 1 Three-dimensional model of TATRA 815 S1 lorry undercarriage and subsidiary ladder frame

\footnotetext{
* Milan Vasko ${ }^{1}$, Bohus Leitner ${ }^{2}$, Milan Saga ${ }^{1}$

${ }^{1}$ KAME, Faculty of Mechanical Engineering, University of Zilina, Slovakia, E-mail: milan.vasko@fstroj.uniza.sk

${ }^{2}$ Faculty of Special Engineering, University of Zilina, Slovakia
} 
The subsidiary ladder frame welded from longitudinal "U" shapes is mounted on the transoms by bolted connections. The "U" shapes are going through all the vehicle length and are interconnected by transoms. A cab, an engine together with a clutch, a sleigh, a reel, bumpers, draw-bar equipment etc. are mounted on transoms, brackets and holders of the subsidiary ladder frame.

\section{Computational Vehicle Model}

The computational finite element model of the TATRA 815 S1 vehicle was built-up in COSMOS/M program in cooperation with TATRA Koprivnice Company. In general, its applied realization consisted of several consequential phases [9]

- geometry model generation,

- definition of elements, their cross-section constants and material characteristics,

- generation of final element mesh,

- definition of boundary conditions,

- setting of acting load,

- computation and verification of obtained results.

Beam elements of the BEAM3D type, mass elements of the MASS type, axial spring boundary elements of the BOUND type together with damping units were used for generation of the finiteelement model of the analyzed TATRA vehicle. The generated model of the TATRA vehicle is presented in Fig. 2.
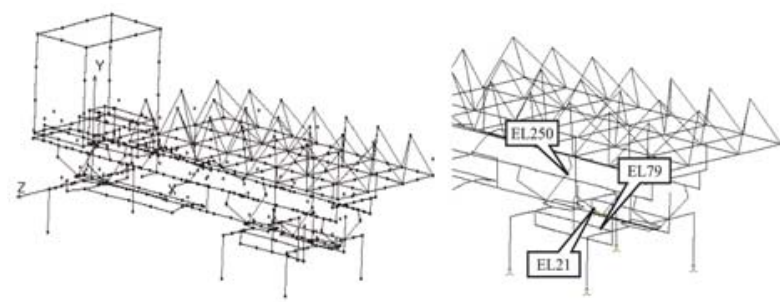

Fig. 2 Finite-element model with definition of chosen vehicle critical parts location

Several elements representing the most exposed parts of the real vehicle construction were determined from the static analysis results - considering a vertical loading character. From these elements, the critical parts from the fatigue life damage accumulation point of view were chosen.

Normal stresses in marginal profile points of elements No. 21,79 and 250 obtained extreme values as ensued from the graphical representation of performed stress analysis results and from the next performed analyses output files [9]. Particular examined elements can be shortly characterized as follows:

- element 21 - part of the vehicle bearing spinal frame, specifically the back bearing tube, construction material - steel STN 41 1523, shape according to Fig. 3a,
- element 79 - part of the formed thick-walled bridge tube of the right central half-axle, construction material - steel STN 41 1523, tube shape according to Fig. 3b,

- element 250 - the longitudinal truss part of the subsidiary bearing frame, approximately in the middle between both back axles, "U" shape $250 \times 100 \times 7$, construction material - steel STN 41 1523, element shape according to Fig. 3c.
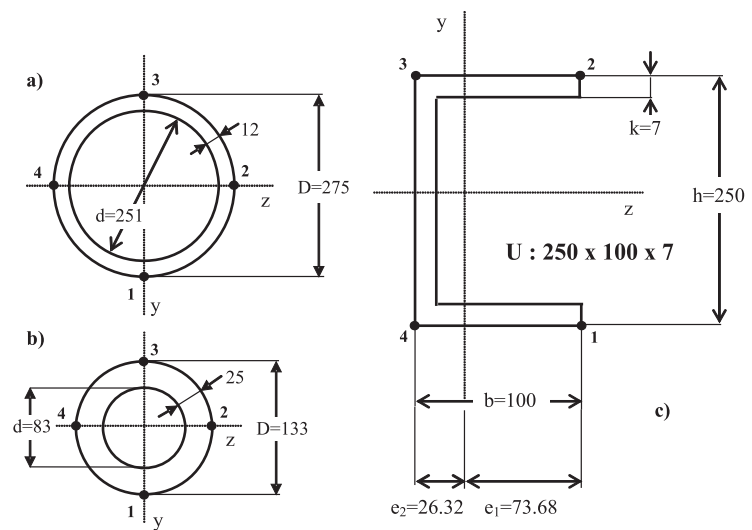

Fig. 3 Cross-sections of the analyzed critical parts

\section{Excitation Model}

The process of kinematics excitation was considered a cooperation of two basic operating factors:

- road surface quality (5 road surface quality classifications and difficult terrain specifications),

- vehicle velocity (4 reference velocities for each road surface quality).

As the excitation generator were applied behaviors of the section's longitudinal height unevenness for different road and terrain surfaces. The method of their acquiring is particularly described for an example in [1,7]. The experimental obtaining of the height unevenness behavior is time and financially demanding. Therefore, the additional realizations needed for the left and right track excitation were obtained by application of the autoregression modeling theory $[6,7]$. Suggested theoretical equipment allows for describing the examined process by means of identified ARMA model and stochastic processes generation with identical statistical characteristics of 1 st and $2^{\text {nd }}$ degree.

The basic process of the road unevenness generation $[3,6,7]$ :

- selection of the road sections with regard to data from road database SSC (5 road surface quality classifications),

- experimental measurement of the longitudinal height unevenness behavior so-called middle longitudinal profiles (exact leveling at an interval of $0.25 \mathrm{~cm}$ ),

- part of the leveling line longitudinal slope and rough unevenness elimination; the random part was extracted, it has a character of a random value of the centered stochastic function and it especially excites the vertical vibration of the vehicle and sig- 
nificantly affects the stochastic vibration of the vehicle's parts during the movement,

- application of the autoregression modeling theory for the suitable ARMA model specification and for generation of new statistically adequate realizations of the stochastic height unevenness behavior on the miscellaneous surface quality.

The behavior of the chosen random kinematics excitation functions for road classification 5 and terrain are shown in Fig. 4.

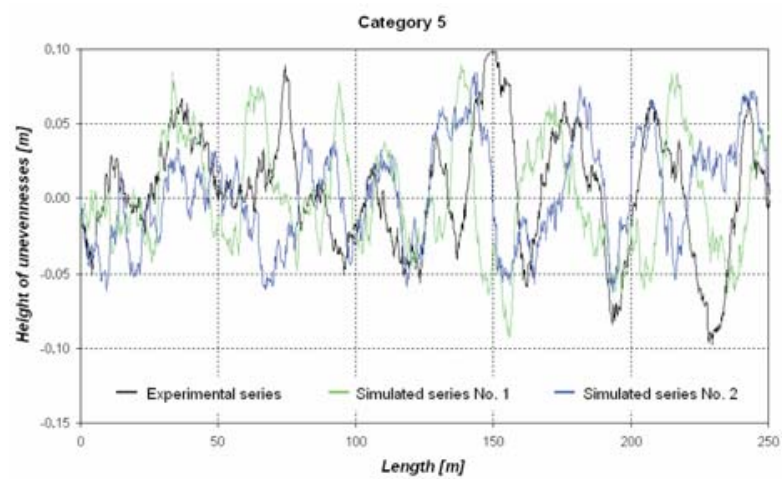

a)

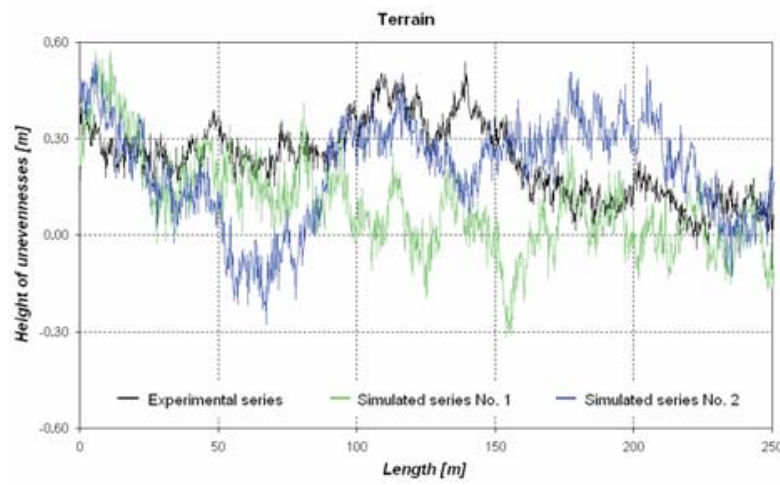

b)

Fig. 4 The random function $-u_{y L}{ }^{(1)}$

a) road classification No. 5, b) terrain

The points where these functions input into the computational model are presented in Fig. 5, where:

$v \quad$ - vehicle speed, $[\mathrm{km} / \mathrm{h}]$,

$L_{1} \quad$ - wheel base No. 1, [m], (see Fig. 5),

$L_{2} \quad$ - wheel base No. 2, [m], (see Fig. 5),

$u_{z L}{ }^{(1)}$ - unevenness of the left rail in a vertical direction for the front axle, [m],

$u_{z P}{ }^{(1)}$ - unevenness of the right rail in a vertical direction for the front axle, $[\mathrm{m}]$,

$u_{z L}{ }^{(2)}$ - unevenness of the left rail in a vertical direction for the $1^{\text {st }}$ back axle, i.e.

$$
u_{z L}^{(2)}(t)=u_{z L}^{(1)}\left(t-\frac{L_{1}}{v}\right),[\mathrm{m}],
$$

$u_{z P}{ }^{(2)}$ - unevenness of the right rail in a vertical direction for the $1^{\text {st }}$ back axle, i.e.

$u_{z P}^{(2)}(t)=u_{z P}^{(1)}\left(t-\frac{L_{1}}{v}\right),[\mathrm{m}]$,

$u_{z L}{ }^{(3)}$ - unevenness of the left rail in a vertical direction for the $2^{\text {nd }}$ back axle, i.e.

$u_{z L}^{(3)}(t)=u_{z L}^{(1)}\left(t-\frac{L_{2}}{v}\right),[\mathrm{m}]$,

$u_{z P}{ }^{(3)}$ - unevenness of the right rail in a vertical direction for the $2^{\text {nd }}$ back axle, i.e.

$u_{z P}^{\left({ }^{(3)}\right.}(t)=u_{z P}^{(1)}\left(t-\frac{L_{2}}{v}\right) .[\mathrm{m}]$,

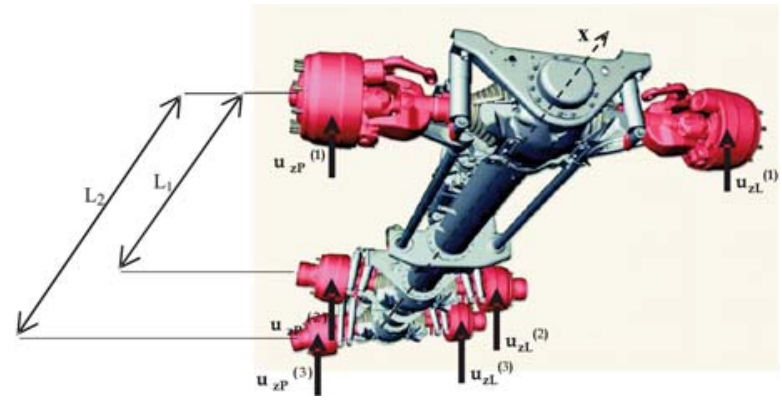

Fig. 5 Identification of the kinematics excitation functions

\section{Computational Fatigue Prediction}

The vehicle operational reliability, in particular its no-failure operation and lifetime is a dominant property for vehicles as a typical representative of the dynamically stressed complex mechanical constructions. These properties continually gain importance. Obviously it relates to raising of the transport velocity, weight reduction of the construction parts, decreasing of the computational safety constants, development and application of the new construction materials, etc. Various vehicles operational failure cause analyses definitely prove that the fatigue process as a dynamic stress consequence assists in nearly all cases.

During the simulation, the dynamic analysis often ends at the modal-spectral construction properties determination, or at its stochastic oscillation solving only from the rigid body oscillation point of view, as for example vehicle vibration, swinging, rolling etc.

If the potential response acquisition is needed too, the problems related to the size can often occur. This means enormous 
requirements for the computer performance and the available operating memory size.

Fatigue life "computation" or rather "estimation" in fact differs from the value achieved during the real machine operation. This is caused mainly by problems related to exact determination of external load characteristic parameters, which affect the construction during operation. Fatigue strength values are not available for specific construction nodes but mainly only for the material samples representing the perfect status, which occurs in real operation conditions only rarely.

Considerations about the problem solution possibilities of the fatigue life estimation can be simply reduced to the four basic tasks:

- selection of the construction critical locations that will become the subject of additional analysis,

- stress determination in the chosen critical locations and its "postprocessing" using the methods suitable for the lifetime estimation

- design or examination of the used construction material properties on the base of the chosen material characteristics,

- selection of a suitable computational procedure - theorem of the fatigue damage cumulation - which effectively joins the material characteristics to the operational stress characteristics. An application result of the suitable fatigue damage cumulation theorem is the lifetime estimation quantified value of the analyzed critical part of the examined construction.

Rajcher's theorem was used for the fatigue damage computations in the identified critical parts of the analyzed vehicle. This theorem defines the fatigue damage in the critical location of the construction part induced per one second and is expressed by the following equation $[2,5]$

$$
D_{s}=\frac{\Gamma\left(\frac{w}{2}+1\right) \cdot\left[2 \cdot \int_{0}^{\infty} f_{w}^{\frac{2}{w}} \cdot S(f) d f\right]^{\frac{w}{2}}}{N_{C} \cdot \sigma_{C}^{w}},
$$

where:

$w \quad-$ exponent of $\mathrm{S} / \mathrm{N}$ curve, [-]

$\sigma_{C} \quad$ - fatigue limit, $[\mathrm{Pa}]$,

$N_{C} \quad$ - limit number of cycles to failure, [-]

$f \quad$ - frequency, $[\mathrm{Hz}]$,

$S(f) \quad$ - spectral power density of the stress loading process, $\left[\mathrm{Pa}^{2} \cdot \mathrm{s}\right]$,

$\Gamma\left(\frac{w}{2}+1\right)$ - gamma function value, [-].

Time until the next failure can be expressed (in hours) as follows

$$
T=\frac{1}{3600 \cdot D_{s}}=\frac{N_{C} \cdot \sigma_{C}^{w}}{3600 \cdot f_{e} \cdot\left(2 \cdot s_{\sigma}^{2}\right)^{\frac{w}{2}}} \cdot \Gamma\left(\frac{w}{2}+1\right) .
$$

It is obvious that all the process can be realized only by means of the computer technique efficient. The approach in practice is that after the import or calculation of the process spectral power density values $S(f)$, the process standard deviation $s_{\sigma}$ and process effective frequency $f_{e}$ of the probability density will be determined.

The practical application of the presented process was realized by the program created in the MATLAB environment. The computational program fatigue.m was used at the fatigue life computational estimation of the construction in the selected critical locations under the chosen characteristics of the lorry operating conditions.

In the application the following material parameters defining the fatigue properties were used:

- slope of $\mathrm{S} / \mathrm{N}$ curve $w$,

- fatigue limit $\sigma_{C}$,

- limit number of cycle $N_{C}$;

which gain the following values for the particular elements:

- for EL 21: $w=5.8, \quad N_{C}=3 \cdot 10^{6}, \quad \sigma_{C}=190 \mathrm{MPa}$,

- for EL 79: $w=5.8, \quad N_{C}=3 \cdot 10^{6}, \quad \sigma_{C}=190 \mathrm{MPa}$,

- for EL 250: $w=5.8, \quad N_{C}=3 \cdot 10^{6}, \quad \sigma_{C}=240 \mathrm{MPa}$.

The value $\sigma_{C}$ was during the process corrected according to the stress average value and also according to factors affecting the fatigue limit (shape, size, treatment quality, stress concentration in the score, etc.).

From the influence analysis of the chosen operational condition characteristics it was determined that the impact of the roadway surfaces from the $1^{\text {st }}$ to $4^{\text {th }}$ class on the TATRA vehicle parts fatigue damage level is nearly negligible. Therefore, only a simulated loading process generated only from the stress behavior originating during the vehicle operation on the $5^{\text {th }}$ class roadways and in the terrain was further applied.

On the base of the statistically determined percentage expression of the analyzed vehicle operation particular mode appearance (Tab. 1), the TATRA critical vehicle parts operation stress processes were created as the implication of the operation loads effect evoked by the analyzed lorry operation on the $5^{\text {th }}$ class roadways and in the terrain.

Percentage ratio estimation of the selected

Tab. 1.

vehicle operation modes occurrence

\begin{tabular}{|l|c|c|c|c|c|c|c|c|}
\hline & \multicolumn{4}{|c|}{$5^{\text {th }}$ class } & \multicolumn{5}{c|}{ terrain } \\
\hline Velocity [km/h] & 20 & 40 & 60 & 80 & 10 & 20 & 30 & 40 \\
\hline Estimation ratio [\%] & 10 & 10 & 10 & 10 & 15 & 20 & 15 & 10 \\
\hline
\end{tabular}

Obtained stresses behavior in the particular critical elements of the TATRA vehicle construction were constituted by 40.000 discrete values representing roadway or terrain distance with the overall length of $10 \mathrm{~km}$. The chosen time stress response is presented in Fig. 6.

The influence of the vehicle movement velocity on the resulting level of the middle axle dynamic stress during the drive under 
the terrain conditions is presented in Fig. 7. Behaviors of the centered stresses part in the bearing beam middle section at the velocity of $80 \mathrm{~km} / \mathrm{h}$ on the road segments with different qualities are presented.

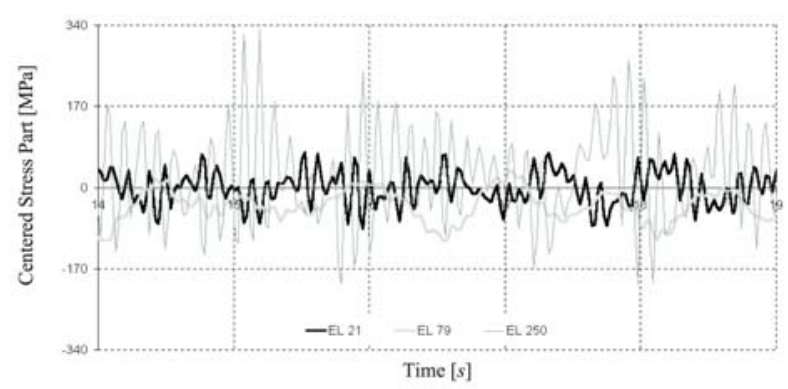

Fig. 6 Time section of the centered stress part behavior at the terrain drive $(v=40 \mathrm{~km} / \mathrm{h})$

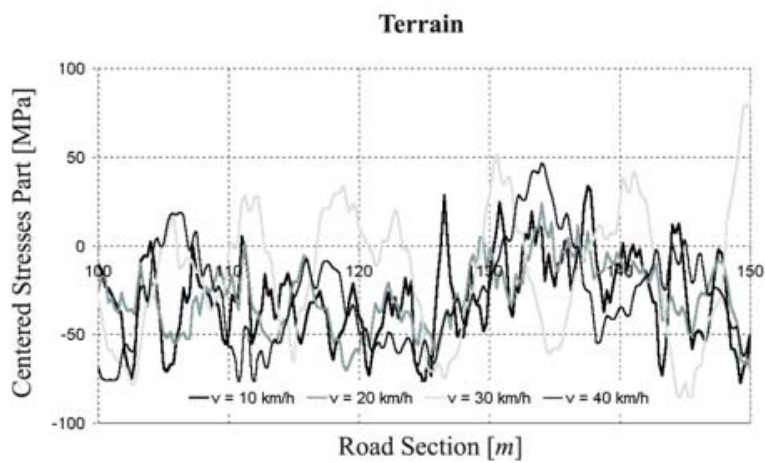

a)
The selected power spectral stress density behavior in the analyzed elements under the chosen velocity and specific roadway class are for the illustration displayed in Fig. 8.

These stress PSD behaviors in all three determined critical construction locations were used as inputs for the fatigue.m program. The critical vehicle parts lifetime estimations were its output. The obtained fatigue life estimations of the particular critical elements are listed in Tab. 2.

Estimated fatigue life values of the vehicle critical

Tab. 2. parts during its real operating employment

\begin{tabular}{|l|l|l|l|l|l|l|}
\hline Element & EL 21 & EL 79 & EL 250a & EL 250b & EL 250c & EL 250d \\
\hline $\begin{array}{l}\text { Lifetime } \\
\text { Estimation } \\
{[\mathrm{h}]}\end{array}$ & 27251 & 19095 & 10743 & 11961 & 595720 & 66133 \\
\hline
\end{tabular}

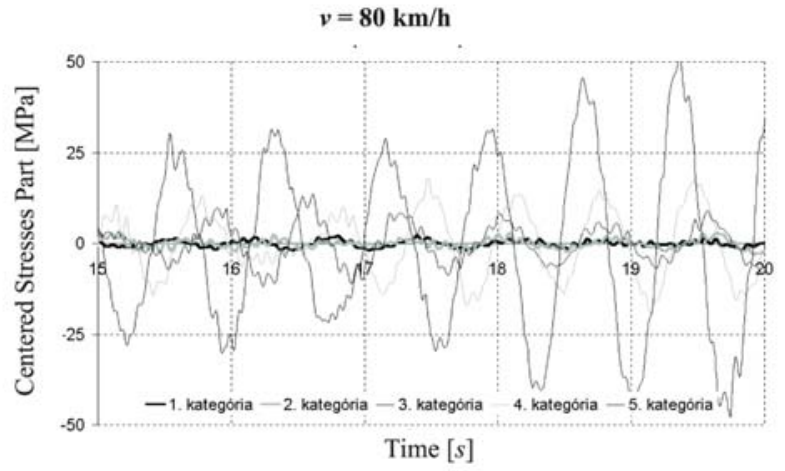

b)

Fig. 7 a) Influence of the surface quality on the centered stresses part in EL 21

b) Influence of the movement velocity on the centered stresses part in EL 79
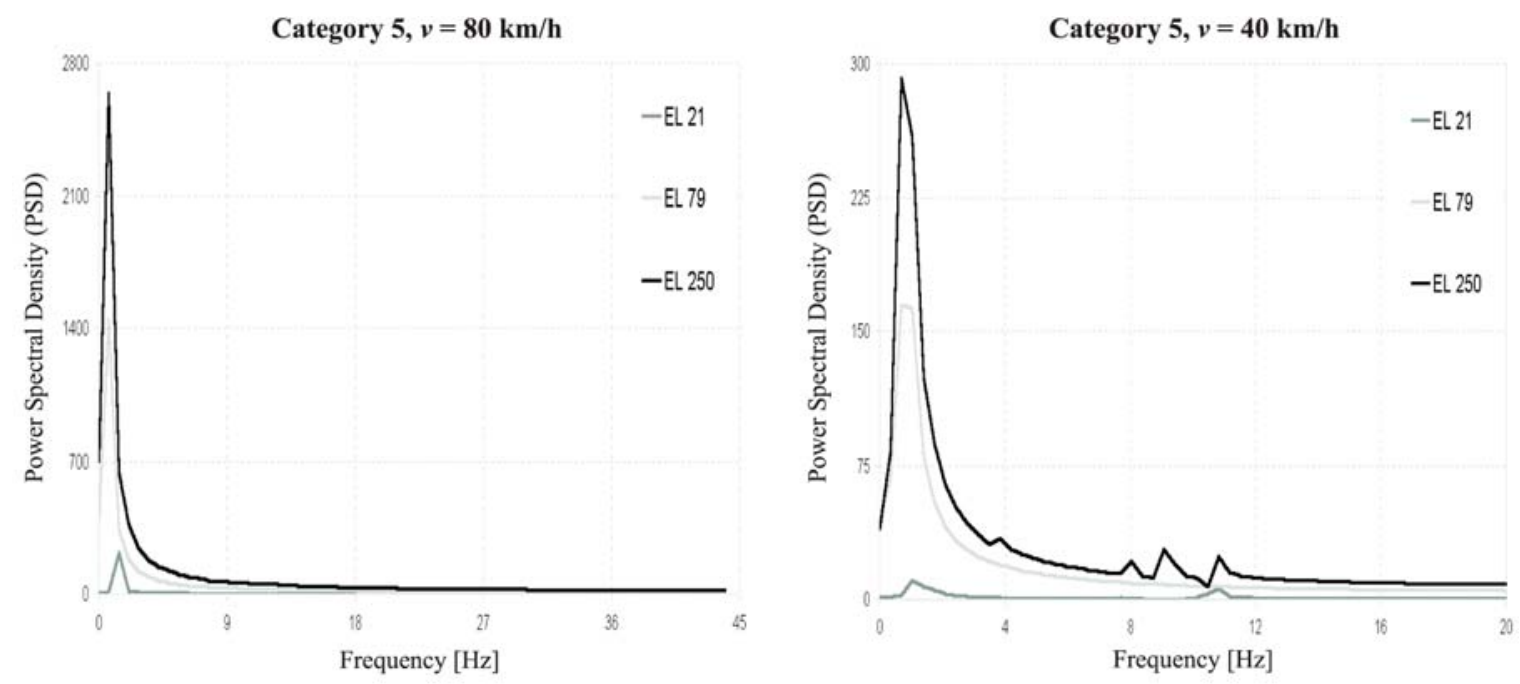

Fig. 8 Stress PSD in the critical elements at the selected surface quality and velocity 
On the base of the performed analyses and comparisons it is possible to state that according to the evaluation of the selected operation condition characteristics influence on the dynamic stress level of the transport vehicle construction parts, both examined characteristics of the working transport vehicle (roadway and terrain undulation and operational velocity) have a significant influence on the stress of the vehicle critical parts.

It is obvious that it was not possible to analyze all the actuating characteristics and factors of the typical lorry operating conditions. Therefore, it is necessary to understand the presented paper as a contribution to the solution of the problem related mainly to the operational processes modeling and computer simulation. Behaviors of particular stress parts and their extreme values in the critical points of the vehicle construction are significantly determined by the chosen vehicle operational velocity $[4,6,8]$.

\section{Conclusion}

Performed calculations confirmed that the analyzed TATRA lorry construction was dimensioned sufficiently also from the fatigue damage point of view. The goal of the paper was mainly:

- to present the methodology of the fatigue life prediction based on the modeling of the most important factors of working conditions,

- to show the possibilities of the available software optimal use based on the FEM in order to obtain the results needed to the fatigue life prediction,

- to verify the suggested computational approach on the specified lorry construction.

\section{Acknowledgements}

This work has been supported by VEGA grants No. $1 / 0125 / 09$ and $1 / 0727 / 10$.

\section{References}

[1] CACKO, J., BILY, M., BUKOVECZKY, J.: Measuring, Evaluation and Simulation of Random Industrial Processes [in Slovak]. In: Veda : Bratislava, SR, 1984.

[2] BALDA, M., SVOBODA, J., FROHLICH, V.: Using Hypotheses for Calculating Fatigue Lives of Parts Exposed to Combined Random Loads. In: Engineering Mechanics 2003, pp.12-15, Svratka, 2003.

[3] DECKY, M.: ISO Standards in Evaluation of Straight Surface Road Process [in Slovak]. In: Anniversary volume Q-2001 Asphalt Roads, SF ZU, Zilina, 2001.

[4] GERLICI, J., LACK, T.: Test Benches Computer Control Software Tools Development. Scientific bulletin, Series C, Vol. XVII, Part II, International Multidisciplinary Conference, 5th edition; North University of Baia Mare : Romania, pp. 181-186, 2003.

[5] RUZICKA, M., HANKE, M., ROST, M.: Dynamic Strength and Lifetime [in Czech]. CVUT : Praha, 1989.

[6] LEITNER, B.: Modelling and Simulation of Transport Machines Working Conditions by Using of Autoregressive Models. In: Academic Journal Mechanics, Transport, Communications, Issue 1/2007, Article No. 0079, VTU Todora Kableskova, Sofia, Bulgaria, pp. 10, www: http://mtc-aj.com/php/welcome.php?lang=gb, 2007.

[7] LEITNER, B.: The Software Support for an Identification of Stochastically Loaded Parts of Mechanically Structures. In: Applied and Computational Mechanics, Vol. 1, No. 2, pp. 515-520, Plzen.

[8] SAGA, M., MEDVECKY, M.: Contribution to Machine Frames Optimization Subjected to Fatigue Damage. In: Machine Design 2009, pp. 177-182, Novi Sad, 2009.

[9] SAGA, M., VASKO, M.: Stress Sensitivity Analysis of the Beam and Shell Finite Elements. In: Communications - Scientific Letters of the University of Zilina, Vol. 11, No. 2, 2009, pp. 5-12. 